\section{Cureus}

Received 09/06/2016

Review began 09/15/2016

Review ended 09/18/2016

Published 09/29/2016

\section{(C) Copyright 2016}

Qadeer et al. This is an open access article distributed under the terms of the Creative Commons Attribution License CC-BY 3.0., which permits unrestricted use, distribution, and reproduction in any medium, provided the original author and source are credited.

\title{
Antibiogram of Medical Intensive Care Unit at Tertiary Care Hospital Setting of Pakistan
}

Aayesha Qadeer ${ }^{1}$, Aftab Akhtar ${ }^{2}$, Qurat Ul Ain ${ }^{3}$, Shoab Saadat ${ }^{4}$, Salman Mansoor ${ }^{5}$, Salman Assad $^{6}$, Wasib Ishtiaq ${ }^{1}$, Abid Ilyas ${ }^{2}$, Ali Y. Khan ${ }^{7}$, Yousaf Ajam ${ }^{8}$

1. Internal Medicine, Shifa International Hospital, Islamabad, PAK 2. Department of Pulmonology \& Critical Care Medicine, Shifa International Hospital, Islamabad, Pakistan 3. Medical Officer, Shifa College of Medicine, Shifa International Hospital, Islamabad, PAK 4. Department of Nephrology, Shifa International Hospital, Islamabad, Pakistan, Portsmouth, GBR 5. Department of Neurology, Shifa International Hospital, Islamabad, Pakistan 6. Internal Medicine, Marshall University School of Medicine, Huntington, USA 7. Department of Internal Medicine \& Infectious Diseases, Shifa International Hospital 8. Shifa International Hospital, Islamabad, Pakistan

$\square$ Corresponding author: Salman Assad, salmanassad91@gmail.com Disclosures can be found in Additional Information at the end of the article

\section{Abstract}

Objective: To determine the frequency of micro-organisms causing sepsis as well as to determine the antibiotic susceptibility and resistance of microorganisms isolated in a medical intensive care unit.

Materials and methods: This is a cross-sectional analysis of 802 patients from a medical intensive care unit (ICU) of Shifa International Hospital, Islamabad, Pakistan over a one-year period from August 2015 to August 2016. Specimens collected were from blood, urine, endotracheal secretions, catheter tips, tissue, pus swabs, cerebrospinal fluid, ascites, bronchoalveolar lavage (BAL), and pleural fluid. All bacteria were identified by standard microbiological methods, and antibiotic sensitivity/resistance was performed using the disk diffusion technique, according to Clinical and Laboratory Standards Institute (CLSI) guidelines. Data was collected using a critical care unit electronic database and data analysis was done by using the Statistical Package for Social Sciences (SPSS), version 20 (IBM SPSS Statistics, Armonk, NY).

Results: Gram-negative bacteria were more frequent as compared to gram-positive bacteria. Most common bacterial isolates were Acinetobacter (15.3\%), Escherichia coli (15.3\%), Pseudomonas aeruginosa (13\%), and Klebsiella pneumoniae (10.2\%), whereas Enterococcus (7\%) and methicillin-resistant staphylococcus aureus (MRSA) (6.2\%) were the two most common gram-positive bacteria. For Acinetobacter, colistin was the most effective antibiotic (3\% resistance). For E.coli, colistin (0\%), tigecycline (0\%), amikacin (7\%), and carbapenems (10\%) showed low resistance. Pseudomonas aeruginosa showed low resistance to colistin (7\%). For Klebsiella pneumoniae, low resistance was seen for tigecycline (0\%) and minocycline (16\%). Overall, ICU mortality was $31.3 \%$, including miscellaneous cases.

Conclusion: Gram-negative infections, especially by multidrug-resistant organisms, are on the rise in ICUs. Empirical antibiotics should be used according to the local unit specific data. Constant evaluation of current practice on basis of trends in multidrug resistance and antibiotic consumption patterns are essential. 


\section{Cureus}

Categories: Internal Medicine, Infectious Disease, Pulmonology

Keywords: resistance, bacteria, antibiogram

\section{Introduction}

Sepsis and septic shock are the leading causes of mortality and morbidity in critical care units. After obtaining samples for cultures, antibiotics are started empirically in intensive care units (ICUs) to cover common pathogens causing sepsis. Early provision of adequate antibiotics improves survival outcomes among critically ill patients with infection. The early use of antibiotics provides effective control of infections; however, at the same time, the use of broadspectrum empiric antibiotics is causing an increasing emergence of antibiotic resistance [1]. A rise in multidrug-resistant bacteria is limiting the available therapeutic options for infections in the ICU and further reducing the likelihood that empiric treatment selections will offer adequate coverage for common ICU pathogens [2].

Over the past two decades, there has been a rapid emergence of multidrug-resistant organisms, which is a major problem in terms of infection control [3]. Although $5 \%$ to $10 \%$ of all patients are treated in intensive care units (ICUs), they constitute about $25 \%$ of all nosocomial infections. The incidence is five to 10 times higher than in general hospital wards [4], which means that nosocomial infections are especially prominent in the ICU [5].

There is a wide diversity between institutions in the prevalence of pathogens and in their antimicrobial susceptibility [6]. The hospital antibiogram is a periodic summary of antimicrobial susceptibilities of local bacterial isolates. Antibiograms are often used by clinicians to assess local susceptibility rates as an aid in selecting an empiric antibiotic therapy and in monitoring resistance trends over time within an institution [7]. Therefore, this study aims to determine the types and frequency of microorganisms causing sepsis as well as to determine the antibiotic susceptibility and resistance of microorganisms isolated in medical ICU.

\section{Materials And Methods}

After approval from the institutional review board (IRB) at Shifa International Hospital, Islamabad Pakistan (approval \#558-006-2016), a cross-sectional study was conducted in which data was collected from patients admitted to the medical intensive care unit over a one-year period from August 2015 to August 2016. Pediatric patients were excluded from the survey. Signed informed consent was obtained from every patient before enrollment in the study. Specimens collected were collected from blood, urine, endotracheal secretions, catheter tips, tissue, pus/pus swabs, cerebrospinal fluid (CSF), ascites, bronchoalveolar lavage (BAL), and pleural fluid (Table 1). All samples were inoculated and incubated for 24 to 48 hours. Colonies were subjected to gram staining and gram-negative and gram-positive organisms isolated.

\begin{tabular}{|c|c|c|c|c|c|c|c|c|c|c|c|c|}
\hline Organisms & $\begin{array}{l}\text { Total Sample }(n= \\
568)\end{array}$ & $\begin{array}{l}\text { Percentage } \\
\text { (\%) }\end{array}$ & Blood & Urine & $\begin{array}{l}\text { Tracheal } \\
\text { Secretions }\end{array}$ & $\begin{array}{l}\text { Catheter } \\
\text { Tip }\end{array}$ & Tissue & $\begin{array}{l}\text { Pus/Pus } \\
\text { Swab }\end{array}$ & $\mathrm{CSF}^{*}$ & Ascites & $B A L^{\star \star}$ & $\begin{array}{l}\text { Pleural } \\
\text { Fluid }\end{array}$ \\
\hline $\begin{array}{l}\text { Acinetobacter } \\
\text { baumanii }\end{array}$ & 87 & 15.3 & 11.6 & 0.6 & 28.0 & 29.7 & 0.0 & 4.2 & 0.0 & 33.3 & 18.2 & 0.0 \\
\hline Burkholderia cepacia & 3 & 0.5 & 0.0 & 0.0 & 1.5 & 0.0 & 0.0 & 0.0 & 0.0 & 0.0 & 0.0 & 0.0 \\
\hline Candida albicans & 47 & 8.3 & 4.5 & 25.3 & 0.0 & 2.7 & 0.0 & 4.2 & 0.0 & 0.0 & 0.0 & 0.0 \\
\hline Candida kefyr & 1 & 0.2 & 0.0 & 0.6 & 0.0 & 0.0 & 0.0 & 0.0 & 0.0 & 0.0 & 0.0 & 0.0 \\
\hline
\end{tabular}




\section{Cureus}

\begin{tabular}{|c|c|c|c|c|c|c|c|c|c|c|c|c|}
\hline Candida krusei & 2 & 0.4 & 0.0 & 1.3 & 0.0 & 0.0 & 0.0 & 0.0 & 0.0 & 0.0 & 0.0 & 0.0 \\
\hline Candida parapsilosis & 2 & 0.4 & 1.8 & 0.0 & 0.0 & 0.0 & 0.0 & 0.0 & 0.0 & 0.0 & 0.0 & 0.0 \\
\hline Candida rugosa & 2 & 0.4 & 0.9 & 0.0 & 0.0 & 2.7 & 0.0 & 0.0 & 0.0 & 0.0 & 0.0 & 0.0 \\
\hline Candida spp. & 27 & 4.8 & 0.0 & 17.1 & 0.0 & 0.0 & 0.0 & 0.0 & 0.0 & 0.0 & 0.0 & 0.0 \\
\hline Candida tropicalis & 23 & 4.0 & 1.8 & 12.7 & 0.0 & 0.0 & 0.0 & 0.0 & 0.0 & 11.1 & 0.0 & 0.0 \\
\hline Enterobacter & 13 & 2.3 & 2.7 & 0.6 & 2.5 & 2.7 & 0.0 & 8.3 & 0.0 & 0.0 & 9.1 & 0.0 \\
\hline Enterococcus & 40 & 7.0 & 17.0 & 7.0 & 0.0 & 13.5 & 14.3 & 12.5 & 0.0 & 0.0 & 0.0 & 0.0 \\
\hline Escherichia coli & 87 & 15.3 & 17.9 & 19.0 & 13.0 & 5.4 & 14.3 & 20.8 & 0.0 & 11.1 & 9.1 & 0.0 \\
\hline Klebsiella pneumoniae & 58 & 10.2 & 9.8 & 5.7 & 13.5 & 16.2 & 14.3 & 0.0 & 0.0 & 22.2 & 0.0 & 100.0 \\
\hline Morganella morganii & 2 & 0.4 & 0.0 & 0.6 & 0.0 & 0.0 & 7.1 & 0.0 & 0.0 & 0.0 & 0.0 & 0.0 \\
\hline Proteus mirabilis & 5 & 0.9 & 0.0 & 0.6 & 1.0 & 2.7 & 7.1 & 0.0 & 0.0 & 0.0 & 0.0 & 0.0 \\
\hline $\begin{array}{l}\text { Pseudomonas } \\
\text { aeruginosa }\end{array}$ & 74 & 13.0 & 10.7 & 5.7 & 19.0 & 13.5 & 7.1 & 8.3 & 50.0 & 11.1 & 45.5 & 0.0 \\
\hline Salmonella typhi & 2 & 0.4 & 1.8 & 0.0 & 0.0 & 0.0 & 0.0 & 0.0 & 0.0 & 0.0 & 0.0 & 0.0 \\
\hline Serratia marcescens & 5 & 0.9 & 0.9 & 0.0 & 0.5 & 0.0 & 7.1 & 8.3 & 0.0 & 0.0 & 0.0 & 0.0 \\
\hline MRSAt & 35 & 6.2 & 4.5 & 0.0 & 11.0 & 0.0 & 14.3 & 12.5 & 0.0 & 11.1 & 18.2 & 0.0 \\
\hline MSSAH & 23 & 4.0 & 5.4 & 0.0 & 6.5 & 0.0 & 7.1 & 12.5 & 0.0 & 0.0 & 0.0 & 0.0 \\
\hline Stenotrophomonas & 6 & 1.1 & 2.7 & 0.0 & 1.0 & 2.7 & 0.0 & 0.0 & 0.0 & 0.0 & 0.0 & 0.0 \\
\hline Streptococcus spp. & 4 & 0.7 & 1.8 & 0.0 & 0.0 & 0.0 & 0.0 & 8.3 & 0.0 & 0.0 & 0.0 & 0.0 \\
\hline $\begin{array}{l}\text { Streptococcus } \\
\text { pneumoniae }\end{array}$ & 8 & 1.4 & 1.8 & 0.0 & 2.5 & 0.0 & 0.0 & 0.0 & 50.0 & 0.0 & 0.0 & 0.0 \\
\hline Trichosporon & 1 & 0.2 & 0.0 & 0.6 & 0.0 & 0.0 & 0.0 & 0.0 & 0.0 & 0.0 & 0.0 & 0.0 \\
\hline VRE $\neq$ & 11 & 1.9 & 2.7 & 2.5 & 0.0 & 8.1 & 7.1 & 0.0 & 0.0 & 0.0 & 0.0 & 0.0 \\
\hline
\end{tabular}

\section{TABLE 1: Source of Samples Taken and Organisms Cultured}

*cerebrospinal fluid (CSF); **bronchoalveolar lavage (BAL); $\nmid$ methicillin-resistant Staphylococcus aureus (MRSA); $\nmid+$ methicillinsensitive Staphylococcus aureus (MSSA); ₹vancomycin-resistant enterococcus (VRE)

Antibiotic susceptibility testing was done by the disk diffusion method according to the Clinical and Laboratory Standards Institute (CLSI) recommendations [8]. The antibiotics used were ampicillin, erythromycin, cloxacillin, amoxicillin/clavulanic acid, amikacin, ceftazidime, cefoperazone/sulbactam, piperacillin/tazobactam, ceftriaxone, imipenem, meropenem, ciprofloxacin, gentamicin, doxycycline, penicillin, colistin, co-trimoxazole, moxifloxacin, chloramphenicol, nitrofurantoin, fosfomycin, minocycline, clindamycin, vancomycin, and linezolid. The zone of inhibition was measured and interpreted as susceptible, intermediate, or 


\section{Cureus}

resistant. Binary logistic regression analysis and Chi-square test $\left(X^{2}\right)$ were done to establish a relationship between the length of ICU stay and mortality.

\section{Results}

Of the total of 802 patients, 454/802 (56.6\%) were males and 348/802 (43.3\%) were females. Age distribution of patients admitted to the ICU is shown in Figure 1.

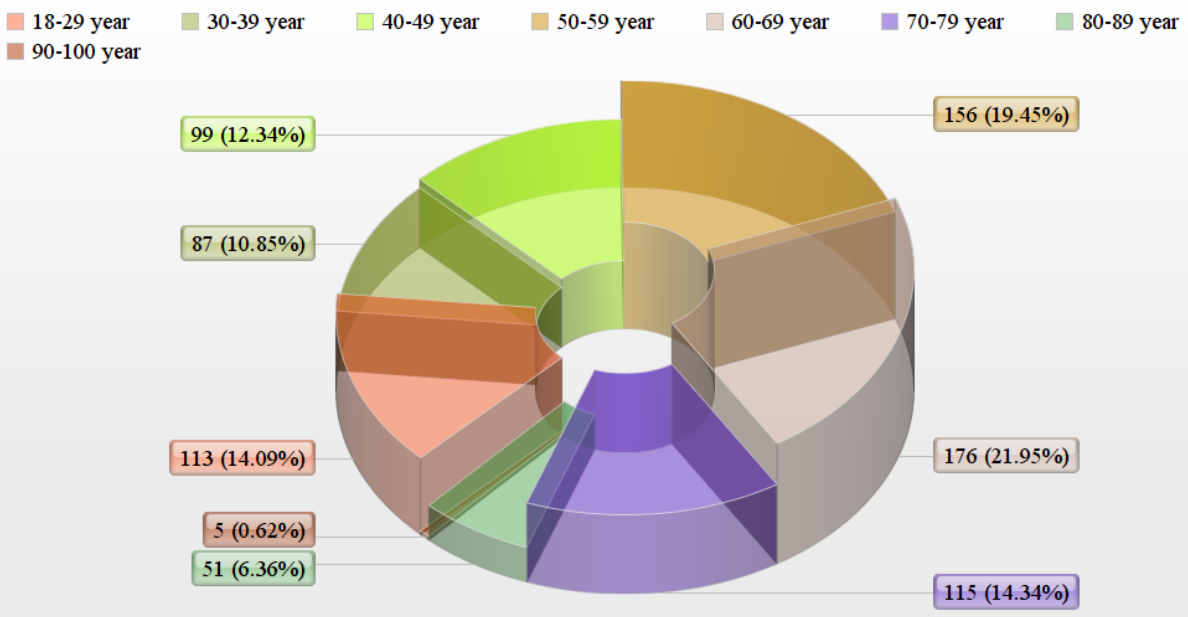

FIGURE 1: Age Distribution of Patients Admitted in the Intensive Care Unit

Three hundred twenty-eight patients (40.89\%) showed positive cultures out of which 265/328 (81.5\%) were bacterial isolates and 64/328 (18.5\%) were Candida albicans. The most frequent isolated gram-negative bacteria were Acinetobacter baumannii (15.3\%), Escherichia coli (15.3\%), Pseudomonas aeruginosa (13\%), and Klebsiella pneumoniae (10.2\%). Other less frequent gram-negative bacteria included Enterobacter (2.3\%), Stenotrophomonas (1.1\%), Proteus mirabilis $(0.9 \%)$, Serratia marcescens $(0.9 \%)$, Burkholderia $(0.5 \%)$, Morganella morganii (0.4\%), and Salmonella typhi (0.4\%) (Figure 2). 


\section{Cureus}

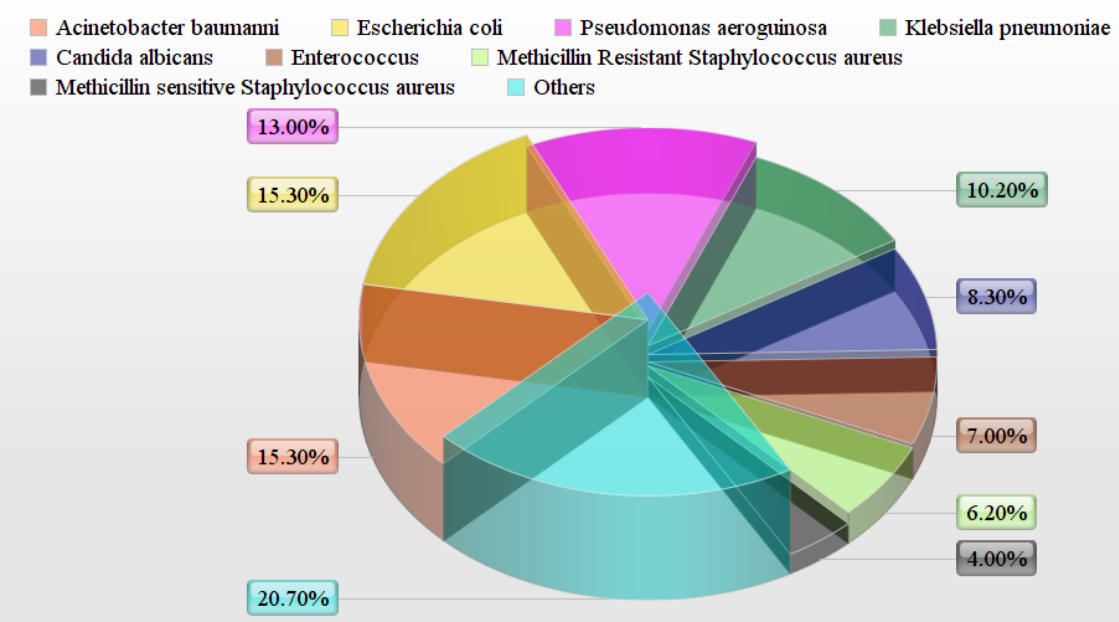

FIGURE 2: Organisms Cultured in Intensive Care Unit Patients

Gram-positive isolates were less frequent as compared to gram-negative isolates and included Enterococcus (7\%), methicillin-resistant Staphylococcus aureus (MRSA) (6.2\%), methicillinsensitive Staphylococcus aureus (MSSA) (4\%), vancomycin-resistant enterococcus (VRE) (1.9\%), Streptococcus pneumonia (1.4\%), and Streptococcus spp (0.7\%). In gram-negative isolates, for Acinetobacter, colistin was the most effective antibiotic (3\% resistance), followed by tigecycline (33\% resistance) and minocycline (36\% resistance). For E.coli, colistin (0\%), tigecycline (0\%), amikacin (7\%), and carbapenems (10\% for both imipenem and meropenem) showed low resistance whereas nitrofurantoin and fosfomycin showed more sensitivity for E.coli urinary tract infections. For Pseudomonas aeruginosa, low resistance was seen for colistin (7\%), ceftazidime (39\%), and amikacin (41\%). For Klebsiella pneumoniae, low resistance was seen for tigecycline (0\%), minocycline (16\%), and colistin (33\%). In gram-positive isolates, for Enterococcus, vancomycin ( $0 \%$ resistance), linezolid ( $0 \%$ resistance), and chloramphenicol (15\% resistance) were the most effective antibiotics, whereas, for MRSA, vancomycin, linezolid, doxycycline, and chloramphenicol showed $0 \%$ resistance and co-trimoxazole showed $17 \%$ resistance (Figure 3). 


\section{Cureus}

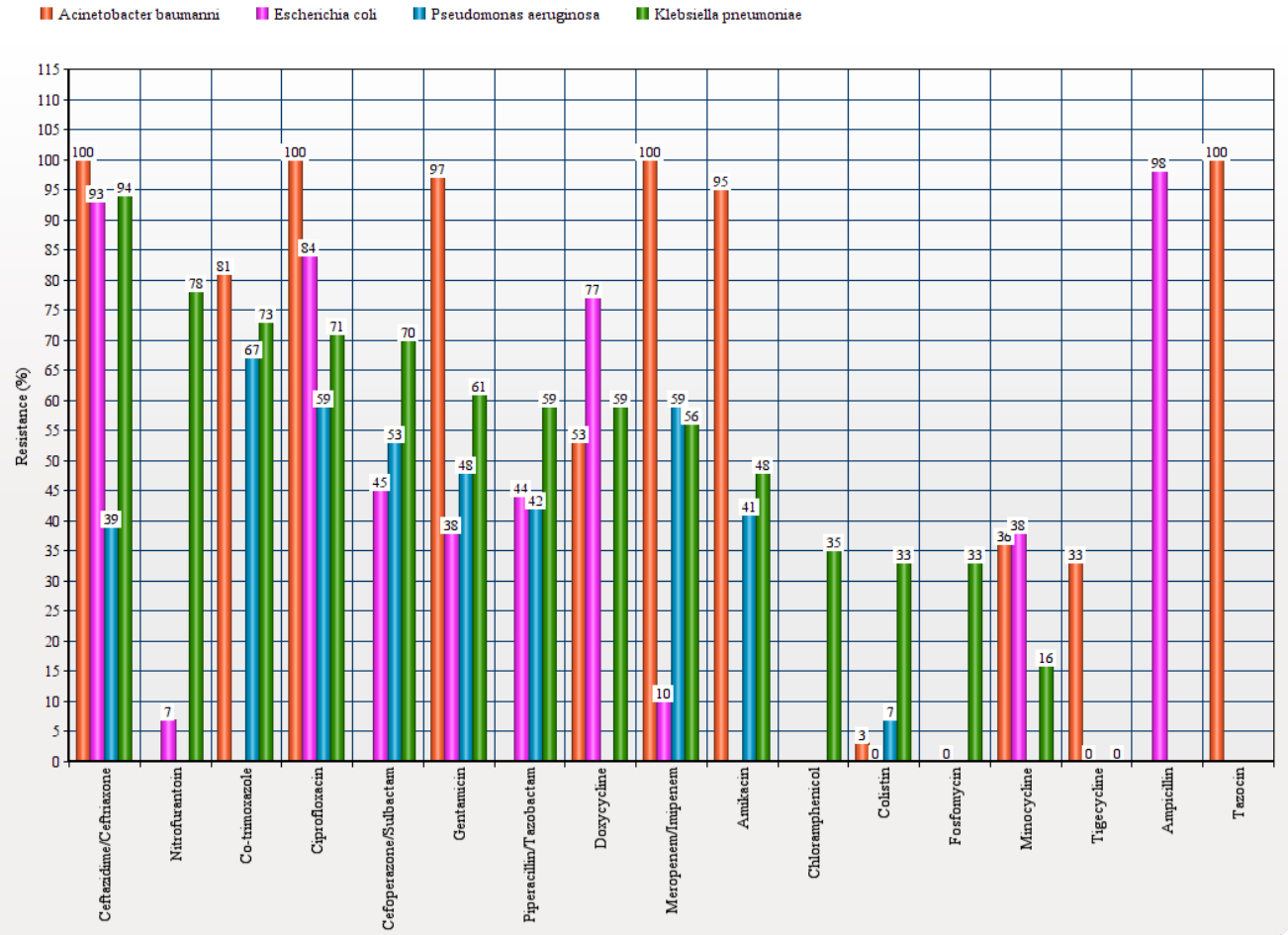

FIGURE 3: Spectrum of Antibiotic Resistance to Bacteria

The length of ICU stay has a negative linear relationship with final outcome in terms of either transferring outpatient with stable vitals or mortality $\left(r=-0.129, X^{2}: \mathrm{p}=0.0001\right)$ (Table 2).

\begin{tabular}{|c|c|c|c|c|c|c|c|c|c|}
\hline \multirow{2}{*}{ Outcome } & \multicolumn{6}{|c|}{ Length of ICU Stay } & \multirow{2}{*}{ Total } & \multirow{2}{*}{$\begin{array}{l}\text { Pearson Correlation } \\
\text { Coefficient (r) }\end{array}$} & \multirow{2}{*}{$\begin{array}{l}\text { X2: } p- \\
\text { value }\end{array}$} \\
\hline & $\begin{array}{l}1-3 \\
\text { days }\end{array}$ & $\begin{array}{l}\text { 4-6 } \\
\text { days }\end{array}$ & $\begin{array}{l}7-9 \\
\text { days }\end{array}$ & $\begin{array}{l}10-12 \\
\text { days }\end{array}$ & $\begin{array}{l}13-15 \\
\text { days }\end{array}$ & $\begin{array}{l}\geq 16 \\
\text { days }\end{array}$ & & & \\
\hline $\begin{array}{l}\text { Transferred } \\
\text { out }(n)\end{array}$ & 286 & 159 & 57 & 26 & 9 & 14 & 551 & \multirow{3}{*}{-0.129} & \multirow{3}{*}{0.0001} \\
\hline Expired (n) & 180 & 43 & 12 & 7 & 4 & 5 & 251 & & \\
\hline Total & 466 & 202 & 69 & 33 & 13 & 19 & 802 & & \\
\hline
\end{tabular}

TABLE 2: Cross-Tabulation Analysis of Outcome and Length of ICU stay

\section{Discussion}

The use of antibiograms to help select empirical antibiotic therapy for suspected infection with likely or known pathogens is a well-established practice. In our study, out of the total bacterial isolates, gram-negative bacteria were more prevalent than were gram-positive bacteria. This predominance of gram-negative bacteria is in concordance with the findings of the similar 
study conducted in 2012 (Al-Jawady, et al.), which may be due to their wide prevalence in the hospital environment. In addition, their frequent resistance to antibiotics may play a role in their persistence and spread [9].

The predominant gram-negative isolates in our study were Acinetobacter and E. coli, whereas the study done by Rajan and Rao showed Klebsiella as the most predominant organism [10]. In a similar study, Ziab, et al. [11] reported Pseudomonas aeruginosa as the predominant gramnegative bacilli being isolated from the ICU, whereas E. coli was the most common gramnegative bacillus reported in studies done by Al-Jawady, et al. and Morfin-Otero, et al. [9, 12]. The most frequent isolate from urine was found to be E. coli in our study. This is comparable to studies conducted by Rajan, et al., Kritupanta, et al., and Sankarankutty, et al. [10, 13-14]. Acinetobacter was most predominant isolate from the respiratory tract in our study. A similar study conducted by Pradhan, et al. showed Acinetobacter to be the most frequent microorganism in respiratory tract [15]. Klebsiella was the most reported organism isolated in the respiratory tract in studies done by Rajan, et al. and Patel, et al. [10, 16].

Our study shows a very high prevalence of carbapenem resistance among Acinetobacter (100\%). A similar study conducted by Mumtaz, et al. [17] has reported 79\% resistance to imipenem, while Rajan, et al. [10] showed 52\% carbapenem resistance among Acinetobacter. In our study, Acinetobacter was highly resistant to third generation cephalosporins (100\% ceftazidime), aminoglycosides (97\% gentamicin and 95\% amikacin), and fluoroquinolones (100\% ciprofloxacin and moxifloxacin). The most effective drug was colistin, which showed 3\% resistance in our study. Similar results of colistin effectiveness against Acinetobacter were seen in the study by Rajan, et al. [10], while work published by Hasan, et al. [18] showed that tigecycline was the most effective antibiotic against Acinetobacter. Our study showed 33\% tigecycline resistance to this bacterium. E. coli, in our study, showed high resistance to third generation cephalosporins (93\% ceftazidime and 90\% ceftriaxone); similarly, more than 90\% E. coli were found to be resistant to third generation cephalosporin by Mohammadi-Mehr, et al.

[19]. Carbapenem resistance was as low as $10 \%$ in our study. Almost similar results reported by Aysen, et al. [20] showed 13.1\% E. coli resistance to imipenem. Gunjal, et al. [21] reported $28.10 \%$ of E. coli isolates were resistant to amikacin and $48.20 \%$ resistance to gentamicin, whereas we found $7 \%$ and $38 \%$ resistance to amikacin and gentamicin, respectively. Fosfomycin, colistin, and tigecycline showed no resistance in E. coli strains.

In our study, Pseudomonas showed significant resistance to carbapenems (59\% imipenem/meropenem), whereas a study published by Rakhee, et al. [22] showed $20.8 \%$ resistance to imipenem and a study published by Rajan, et al. [10] showed $12.9 \%$ carbapenem resistance to Pseudomonas. Pseudomonas also showed high resistance to third generation cephalosporins (53\% cefoperazone/sulbactam and 39\% to ceftazidime) and aminoglycosides (48\% gentamicin and $41 \%$ amikacin) in our study. Radji, et al. showed $60.9 \%$ resistance to ceftriaxone and found that amikacin was the most effective antibiotic against Pseudomonas with $15.6 \%$ resistance [23]. We found colistin to be the most effective antibiotic against Pseudomonas with only $7 \%$ resistance.

Klebsiella, the fourth most common microorganism of our study, showed high carbapenem resistance (56\% meropenem and 55\% imipenem), whereas Sheth, et al. [24] showed 100\% sensitivity to carbapenems and Rajan, et al. [10] documented 28.13\% carbapenem resistance. In our study, a high pattern of resistance was seen with third generation cephalosporins (94\% ceftazidime, $82 \%$ ceftriaxone, and 70\% cefoperazone/sulbactam) and aminoglycosides (61\% gentamicin, $48 \%$ amikacin). Gunjal, et al. have reported $60 \%$ resistance to amikacin and $80 \%$ resistance to gentamicin [21]. Colistin was found to be the most effective antibiotic for multidrug-resistant (MDR) Acinetobacter, E. coli, and Pseudomonas, whereas tigecycline was found to be the effective antibiotic against multidrug-resistant Klebsiella. 


\section{Limitations}

External validation of this cross-sectional survey cannot be determined. The analysis is based on single centre experience and associated comorbidities have not been taken into account. These comorbidities might be contributing towards Neyman's (prevalence) bias. The confounding factors, including age and gender, might affect overall ICU mortality.

\section{Conclusions}

Multidrug-resistant gram-negative infections are on the rise in ICUs and are one of the contributory factors in the increase in overall ICU morbidity and mortality. Resistance to important antibiotic groups, including quinolones, piperacillin-tazobactam, and carbapenems, has increased substantially over the past few years. It is suggested that empiric antibiotics should be used according to the local ICU unit antibiograms. A constant evaluation of current practices on the basis of trends in multidrug resistance and antibiotic consumption patterns is essential.

\section{Additional Information \\ Disclosures}

Human subjects: Consent was obtained by all participants in this study. Institutional Review Board at Shifa International Hospital, Islamabad Pakistan issued approval \#558-006-2016. Animal subjects: All authors have confirmed that this study did not involve animal subjects or tissue. Conflicts of interest: In compliance with the ICMJE uniform disclosure form, all authors declare the following: Payment/services info: All authors have declared that no financial support was received from any organization for the submitted work. Financial relationships: All authors have declared that they have no financial relationships at present or within the previous three years with any organizations that might have an interest in the submitted work. Other relationships: All authors have declared that there are no other relationships or activities that could appear to have influenced the submitted work.

\section{References}

1. Kaki R, Elligsen M, Walker S, Simor A, Palmay L, Daneman N: Impact of antimicrobial stewardship in critical care: a systematic review. J Antimicrob Chemother. 2011, 66:1223-30. 10.1093/jac/dkr137

2. Randhawa V, Sarwar S, Walker S, Elligsen M, Palmay L, Daneman N: Weighted-incidence syndromic combination antibiograms to guide empiric treatment of critical care infections: a retrospective cohort study. Crit Care. 2014, 18:R112. 10.1186/cc13901

3. Brust K, Evans A, Plemmons R: Tigecycline in treatment of multidrug-resistant Gramnegative bacillus urinary tract infections: a systematic review. J Antimicrob Chemother. 2014, 69:2606-10. 10.1093/jac/dku189

4. Al Johani SM, Akhter J, Balkhy H, El-Saed A, Younan M, Memish Z: Prevalence of antimicrobial resistance among gram-negative isolates in an adult intensive care unit at a tertiary care center in Saudi Arabia. Ann Saudi Med. 2010, 30:364-69.

5. Dasgupta S, Das S, Chawan NS, Hazra A: Nosocomial infections in the intensive care unit: Incidence, risk factors, outcome and associated pathogens in a public tertiary teaching hospital of Eastern India. Indian J Crit Care Med. 2015, 19:14-20. 10.4103/0972-5229.148633

6. Fridkin SK: Increasing prevalence of antimicrobial resistance in intensive care units . Crit Care Med. 2001, 29:N64-68. 10.1097/00003246-200104001-00002

7. Joshi S: Hospital antibiogram: a necessity. Indian J Med Microbiol. 2010, 28:277-80. 10.4103/0255-0857.71802

8. Wolfensberger A, Sax H, Weber R, Zbinden R, Kuster SP, Hombach M: Change of antibiotic susceptibility testing guidelines from CLSI to EUCAST: influence on cumulative hospital antibiograms. PLoS One. 2013, 8:e79130. 10.1371/journal.pone.0079130

9. Al-Jawady Z, Al-Habib HM: Antibiogram profiles of bacterial isolates from intensive care units 
in Mosul Teaching Hospitals. Raf J Sci. 2012, 23:52-59.

10. Rajan R, Rao AVR: Antibiogram of gram-negative bacterial isolates from intensive care unit at a tertiary care hospital. IJAR. 2016, 6:344-47.

11. Al-Ahmadey ZZ, Mohamed SA: Antimicrobial susceptibility pattern of bacterial isolates in the intensive care unit of Al-Ansar Hospital,Saudi Arabia. Eur J Adv Res Bio Life Science. 2013, 1:17-26.

12. Morfin-Otero R, Tinoco-Favila JC, Sader HS, Salcido-Gutierrez L, Perez-Gomez HR, GonzalezDiaz E, Petersen L, Rodriguez-Noriega E: Resistance trends in gram-negative bacteria: surveillance results from two Mexican hospitals, 2005-2010. BMC Res Notes. 2012, 5:277. 10.1186/1756-0500-5-277

13. Panta K, Ghimire P, Rai SK, Mukiya RK, Singh RN, Rai G: Antibiogram typing of gram negative isolates in different clinical samples of a tertiary care hospital. Asian J Pharm Clin Res. 2013, 6:153-56.

14. Sankarankutty J, Kaup S: Distribution and antibiogram of gram negative isolates from various clinical samples in a teaching hospital Tumkur. Sch J App Med Sci. 2014, 2:927-31.

15. Pradhan NP, Bhat SM, Ghadage DP: Nosocomial infections in the medical ICU: a retrospective study highlighting their prevalence, microbiological profile and impact on ICU stay and mortality. J Assoc Physicians India. 2014, 62:18-21.

16. Patel BV, Patel PG, Raval PN, Patel MH, Patel PH, Vengad MM: Bacteriological profile and antibiogram of gram negative organisms isolated from medical and neurology intensive care units with special reference to multidrug resistant organisms. Natl J Med Res. 2012, 3:335-37.

17. Khan MA: Bacterial spectrum and susceptibility patterns of pathogens in ICU and IMCU of a Secondary Care Hospital in Kingdom of Saudi Arabia. Int J Pathol. 2012, 10:64-70.

18. Hasan B, Perveen K, Olsen B, Zahra R: Emergence of carbapenem-resistant Acinetobacter baumannii in hospitals in Pakistan. J Med Microbiol. 2014, 63:50-55. 10.1099/jmm.0.0639250

19. Mohammadi-Mehr M, Feizabadi M: Antimicrobial resistance pattern of Gram-negative bacilli isolated from patients at ICUs of Army hospitals in Iran. Iran J Microbiol. 2011, 3:26-30.

20. Bayram A, Balci I: Patterns of antimicrobial resistance in a surgical intensive care unit of a university hospital in Turkey. BMC Infect Dis. 2006, 6:155. 10.1186/1471-2334-6-155

21. Gunjal P, Gunjal S, Kher S: A cross-sectional study to determine the profile and antibiotic resistance pattern of gram negative bacilli isolated from intensive care unit patients in a tertiary care hospital in Ahmednagar, Maharastra. Int J Biomed Adv Res. 2012, 3:281-84. 10.7439/ijbar.v3i5.375

22. Raakhee T, Rao US: Prevalence and resistance pattern of pseudomonas strains isolated from ICU patients. Int J Curr Microbiol App Sci. 2014, 3:527-34.

23. Radji M, Fauziah S, Aribinuko N: Antibiotic sensitivity pattern of bacterial pathogens in the intensive care unit of Fatmawati Hospital, Indonesia. Asian Pac J Trop Biomed. 2011, 1:39-42. 10.1016/S2221-1691(11)60065-8

24. Sheth KV, Patel TK, Malek SS, Tripathi CB: Antibiotic sensitivity pattern of bacterial isolates from the intensive care unit of a tertiary care hospital in India. Trop J Pharm Res. 2012, 11:991-99. 10.4314/tjpr.v11i6.17 